\title{
An Analogue-Beam Splitting Approach for MmWave D2D Multicast Channel
}

\author{
Lifu Liu, Yi Ma, Na Yi, and Rahim Tafazolli \\ Institute for Communication Systems (ICS) \\ University of Surrey, Guildford, England, GU2 7XH \\ Email: (lifu.liu, y.ma, n.yi, r.tafazolli)@ surrey.ac.uk
}

\begin{abstract}
Considering a densely populated area where a mobile device, with a single RF chain, shares its message with a set of mobile devices through narrowband mmWave channel, an analogue-beam splitting approach is proposed to achieve a good capacity and coverage trade-off. The proposed approach aims at maximizing the capacity of the mmWave multicast channel through antenna-element grouping and adaptive phase shifting, which takes into account of the inter-beam interference. When receivers are randomly distributed on a circle centered at the transmitter, according to the uniform distribution, it is found that the impact of inter-beam interference on the channel capacity can be negligibly small, and thus the analoguebeam splitting approach can be largely simplified in practice. Computer simulations are carried out to elaborate our theoretical study and demonstrate considerable advantages of the proposed analogue-beam splitting approach.
\end{abstract}

\section{INTRODUCTION}

Mobile operators are currently facing the technical challenge for delivering data and voice services to their customers in densely populated areas such as stadium hot-spot, airport, business center, shopping mall, tube station and many others [1]. Investment in spectrum license or network infrastructure (such as dense MIMO networks) might be a possible approach to handle the challenge however at significant pay for CAPEX and OPEX. A cost-effective approach could be by allowing for mobile devices to form direct communications (i.e. D2D), which can be particularly useful for network traffic offloading when users in a local area share common interest in mobile contents [2], [3]. Despite, the existence of many D2D links renders the network performance very interference limited. Such motivates the use of mmWave band (lower license cost or unlicensed spectrum) for local D2D communications [4], while the access network remains on the conventional radio frequencies (typically below $6 \mathrm{GHz}$ ) [5].

Current mmWave technology is optimized for fixed point-to-point $(\mathrm{P} 2 \mathrm{P})$ communications due to its nature of using directional signal transmission for the purpose of coverage extension [6]. Moreover, mobile devices are often equipped with a single RF-chain for the sake of cost efficiency [7]. Those facts rather limit the multiuser capability of D2D communications, which are however critical for the spectral efficiency when a mobile user wants to share its contents with a number of local mobile users. A straightforward approach is by allowing the transmitter to communicate with its desired receivers through beam steering in the time-division duplexing (TDD) manner [8]. By this means, the channel capacity decreases linearly with the number of receivers. Alternatively, it is possible to conduct D2D multicast through analogue multiuser beamforming. Given a single RFchain, the radio beams are formed through analogue phase-shifting array [9]. Thanks to the use of large excess of transmit-antenna elements over receivers, antenna elements can be divided into several groups with each forming an analogue beam to serve a receiver; such is called the analogue-beam splitting approach in this paper.

The use of analogue-beam splitting for multicast could face two problems. One is the inter-beam interference which can be either constructive or destructive depending on the phase difference between the signal and the interference. The other is the fundamental trade-off between the radio coverage and the user capacity; as increasing beams often mean reducing signal-to-noise ratio (SNR). In this paper, we investigate these two problems through both theoretical analysis and computer simulations.

Our investigation starts from the theoretical study of the mmWave multicast channel capacity and the coverage [10]. The capacity-coverage trade-off is mathematically established incorporating the correlation between receivers. Given the beamforming design for multicast a NP-hard problem, an analogue-beam splitting approach is developed as a sub-optimum solution, with its feasibility condition and design principle being mathematically justified. It is shown that the receiver correlation introduces inter-beam interference, which could be either constructive or destructive. Considering the use of large excess of transmit antenna-elements over receivers, it is found that the inter-beam interference is negligible; and such largely simplifies the analogue-beam splitting approach. In terms of the multicast channel capacity, it is shown that the proposed analogue-beam splitting approach significantly outperforms the TDD beam-steering approach by $2-3$ fold. This result is elaborated through 
computer simulations with a practical mmWave channel model.

\section{Modeling D2D Multicast Over MmWave CHANNEL}

\section{A. MmWave D2D Multicast Model}

Consider a two-dimensional (2D) wireless communication model, where a mobile device shares a common message to its desired mobile receivers located on a circle centered by the transmitter; see Fig. 1. The transmitter-receiver separation is the radius of the circle denoted by $d$. Such a simplified system model is widely used for the link-level study [4], and thus it is of our interest. Furthermore, it is assumed that all mobile devices have a single RF-chain associated with $N$ antenna elements either for the transmission or reception.

Denote $s$ to be the information-bearing symbol sent by the transmitter. Prior to transmission, a $(N) \times(1)$ beamforming vector $\mathbf{b}$ is employed to map $s$ onto the antenna elements, with the transmitted waveform represented by a $(N) \times(1)$ vector

$$
\mathbf{x}=\mathbf{b} s
$$

It is worthwhile to note that the beamforming vector b is generated by the RF phase-shift array, and thus each element of $\mathbf{b} \triangleq\left[b_{0}, \ldots, b_{N-1}\right]^{T}$ has an unity gain: $\left|b_{n}\right|=1, \forall n \in[0, N-1]$.

The waveform $\mathrm{x}$ goes through the mmWave channel between the transmitter and the $k^{\text {th }}$ receiver [11]

$$
\mathbf{H}_{k}=\sum_{l=0}^{L-1} g_{l} \boldsymbol{\alpha}_{\mathrm{r}}\left(\phi_{k, l}\right) \boldsymbol{\alpha}_{\mathrm{t}}^{T}\left(\theta_{k, l}\right), k \in[0, K-1]
$$

where $L$ stands for the number of multipath, $g_{l}$ for the gain of the $l^{\text {th }}$ propagation path, $K$ for the number of receivers, $\theta_{k, l}$ for the angle of departure (AoD), $\phi_{k, l}$ for the angle of arrival (AoA) with $\theta_{k, l}, \phi_{k, l} \in\left[-\frac{\pi}{2}, \frac{\pi}{2}\right]$, and the superscript $[\cdot]^{T}$ stands for the matrix/vector transpose. The vectors $\boldsymbol{\alpha}_{\mathrm{r}}\left(\phi_{k, l}\right)$ and $\boldsymbol{\alpha}_{\mathrm{t}}\left(\theta_{k, l}\right)$ are defined by: $\boldsymbol{\alpha}_{\mathrm{r}}\left(\phi_{k, l}\right) \triangleq \frac{1}{\sqrt{N}}\left[1, e^{j \beta}, \ldots, e^{j \beta(N-1)}\right]^{T}$ and $\boldsymbol{\alpha}_{\mathrm{t}}\left(\theta_{k, l}\right) \triangleq$ $\frac{1}{\sqrt{N}}\left[1, e^{j \gamma}, \ldots, e^{j \gamma(N-1)}\right]^{T}$ with $\beta \triangleq \frac{2 \pi q}{\lambda} \sin \left(\phi_{k, l}\right)$ and $\gamma \triangleq \frac{2 \pi q}{\lambda} \sin \left(\theta_{k, l}\right)$, where $\lambda$ is the wavelength, and $q$ the separation between neighboring antenna elements, which is often set to $q=\frac{\lambda}{2}$ for the narrowband mmWave channel. The received waveform at the $k^{\text {th }}$ receiver is given by

$$
\begin{aligned}
y_{k} & =\mathbf{r}_{k}^{T} \mathbf{H}_{k} \mathbf{x}+v_{k}, \quad k=0, \ldots, K-1 \\
& =\mathbf{r}_{k}^{T} \mathbf{H}_{k} \mathbf{b} s+v_{k}
\end{aligned}
$$

where $\mathbf{r}_{k} \triangleq\left[r_{0}, \ldots, r_{N-1}\right]^{T}$ is the receiver beamforimg vector with $\left|r_{n}\right|=1, \forall n \in[0, N-1]$, and $v_{k}$ the white Gaussian noise with zero mean and variance of $\sigma_{v}^{2}$.

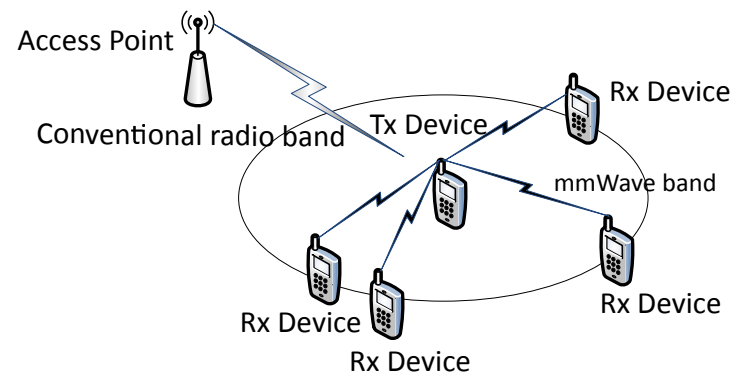

Fig. 1. Illustration of D2D multicast communications over mmWave band.

\section{B. Multicast Capacity}

Given (4), the channel capacity for the transmitter to the $k^{\text {th }}$ receiver link is

$$
C_{k}=\log _{2}(1+\underbrace{\frac{\sigma_{s}^{2}}{\sigma_{v}^{2}}\left|\mathbf{r}_{k}^{T} \mathbf{H}_{k} \mathbf{b}\right|^{2}}_{\triangleq \mathrm{SNR}_{k}})
$$

Since receivers are interested only in the common message, the multicast channel capacity is simply the mincut capacity

$$
C=\min \left(C_{0}, \ldots, C_{K-1}\right)
$$

For the sake of spectral efficiency, the transmitter shall maximize the capacity $C$ by finding the optimal configuration for $\mathbf{b}$

$$
\mathbf{b}^{\star}=\underset{\mathbf{b}}{\arg \max } C(\mathbf{b})
$$

Here we represent the capacity $C$ as a function of $\mathbf{b}$ by assuming the knowledge of $\mathbf{H}_{k}$, whilst the receiver beamforming $\mathbf{r}_{k}$ can be individually optimized at the receiver side. The optimization for (7) is the typical maxmin capacity problem, which is NP hard [12]. Therefore, a sub-optimum approach will be introduced in Sec. III.

\section{Multicast Coverage}

The multicast coverage highly depends on the number of users (i.e. $K$ ) as well as their mutual correlations. When the channels for all receivers are fully correlated (i.e. identical $\mathbf{H}_{k}, \forall k$ ), the coverage issue is equivalent to the $\mathrm{P} 2 \mathrm{P}$ case, where the capacity is directly linked to the transmitter-receiver separation. When the channels are mutually orthogonal (i.e. $\mathbf{H}_{k_{1}} \mathbf{H}_{k_{2}}^{H}=\mathbf{0}, \forall k_{1} \neq k_{2}$, $[\cdot]^{H}$ for the matrix Hermitian transpose), the power of $s$ has to be evenly allocated to each receiver; and this is the worst-case scenario where the signal-to-noise ratio (SNR) degrades linearly with the number of receivers.

In the general case when receivers distribute randomly on the circle, the channel correlation between receivers becomes very random. The correlation between arbitrary 
two receivers is determined by the AoD, and it can be mathematically defined by

$$
\begin{aligned}
\rho\left(k_{1}, k_{2}, l_{1}, l_{2}\right) & \triangleq \boldsymbol{\alpha}_{\mathrm{t}}^{H}\left(\theta_{k_{1}, l_{1}}\right) \boldsymbol{\alpha}_{\mathrm{t}}\left(\theta_{k_{2}, l_{2}}\right) \\
& =\frac{1}{N} \sum_{n=0}^{N-1} \exp \left(j n \pi \Delta\left(k_{1}, k_{2}, l_{1}, l_{2}\right)\right) \\
& =\frac{1-\exp \left(j N \pi \Delta\left(k_{1}, k_{2}, l_{1}, l_{2}\right)\right)}{N\left(1-\exp \left(j \pi \Delta\left(k_{1}, k_{2}, l_{1}, l_{2}\right)\right)\right)}
\end{aligned}
$$

where $\Delta\left(k_{1}, k_{2}, l_{1}, l_{2}\right)=\sin \left(\theta_{k_{2}, l_{2}}\right)-\sin \left(\theta_{k_{1}, l_{1}}\right)$, $k_{1}, k_{2} \in[0, K-1]$ and $l_{1}, l_{2} \in[0, L-1]$. When receivers distribute uniformly on the circle, it can be numerically shown that $\rho\left(k_{1}, k_{2}, l_{1}, l_{2}\right)$, for $l_{1}=l_{2}$, has its amplitude following approximately the exponential distribution with $p(|\rho|<20 \%)>90 \%$, and its phase following linear distribution within the range $(-\pi, 0)$ and $(0, \pi)$ respectively, with the maximal probability (around $3.3 \%$ ) occurring for $\angle \rho$ close to 0 , and the least probability (around $0.05 \%$ ) occurring for $\angle \rho$ close to $-\pi$ or $\pi$. It is worthwhile to note that the numerical result is obtained for the case of $N=32$, and the amplitude of $\rho$ will become smaller given a larger $N$.

Basically, it can be concluded that the channel correlation between receivers is mostly very weak. On the other hand, the phase of $\rho$ varies widely, and it could render the channel correlation either constructive or destructive. In a very optimistic case assuming no destructive correlation, the power allocation for receivers (or equivalently SNR for each receiver) scales with the number of receivers approximately as

$$
\frac{\mathrm{SNR}_{k}}{\mathrm{SNR}_{\mathrm{P} 2 \mathrm{P}}} \approx \frac{1}{K(1-|\rho|)}, k=0, \ldots, K-1
$$

which counts only two-receiver mutual correlation. This result reflects the trade-off between the user capacity (i.e. $K$ ) and the coverage (i.e. SNR). It is clear that the SNR at each receiver degrades still linearly with the number of receivers. Given the correlation as small as $20 \%$ or below, the impact of $\rho$ to the coverage is not considerable particularly for a large $K$.

\section{Analogue-beam Splitting For D2D MmWaVE Multicast}

Given the optimization for (7) a NP-hard problem, we propose an analogue-beam splitting approach as a sub-optimal solution. The proposed approach simply divides the transmit antenna-elements into $K$ groups, with each having $\bar{N}=(N) /(K)$ antenna elements. When $\bar{N}$ is not an integer, there are $(N-\lfloor\bar{N}\rfloor K)$ groups having $(\lfloor\bar{N}\rfloor+1)$ antenna elements, and others having $\lfloor\bar{N}\rfloor$ antenna elements. A receiver with lower SNR is suggested to be put into a group with more antenna elements for the SNR enhancement. For the sake of simplifying our presentation, we assume $\bar{N}$ to be an integer for the rest of the paper.

\section{A. Orthogonal Beam Splitting and Feasibility Condition}

Mathematically, the concept of beam splitting (or antenna-element grouping) is to split the beamforming vector $\mathbf{b}$ into $K$ sub-vectors with each having the size of $(\bar{N}) \times(1)$. The objective is to assure $\mathrm{SNR}_{k}$ to be identical $\forall k \in[0, K-1]$, which means

$$
\left|\mathbf{r}_{k_{1}}^{T} \mathbf{H}_{k_{1}} \mathbf{b}\right|^{2}=\left|\mathbf{r}_{k_{2}}^{T} \mathbf{H}_{k_{2}} \mathbf{b}\right|^{2}, \forall k_{1} \neq k_{2}
$$

Considering the fact of very weak multipath components in the mmWave channel, (11) can be approximately represented by

$$
\begin{gathered}
\frac{\left|\mathbf{r}_{k_{1}}^{T} \boldsymbol{\alpha}_{\mathrm{r}}\left(\phi_{k_{1}}\right) \boldsymbol{\alpha}_{\mathrm{t}}^{T}\left(\theta_{k_{1}}\right) \mathbf{b}\right|^{2}}{\left|\mathbf{r}_{k_{2}}^{T} \boldsymbol{\alpha}_{\mathrm{r}}\left(\phi_{k_{2}}\right) \boldsymbol{\alpha}_{\mathrm{t}}^{T}\left(\theta_{k_{2}}\right) \mathbf{b}\right|^{2}}=1 \\
\Longrightarrow \frac{\left|\mathbf{r}_{k_{1}}^{T} \boldsymbol{\alpha}_{\mathrm{r}}\left(\phi_{k_{1}}\right)\right|^{2}\left|\boldsymbol{\alpha}_{\mathrm{t}}^{T}\left(\theta_{k_{1}}\right) \mathbf{b}\right|^{2}}{\left|\mathbf{r}_{k_{2}}^{T} \boldsymbol{\alpha}_{\mathrm{r}}\left(\phi_{k_{2}}\right)\right|^{2}\left|\boldsymbol{\alpha}_{\mathrm{t}}^{T}\left(\theta_{k_{2}}\right) \mathbf{b}\right|^{2}}=1
\end{gathered}
$$

Given $\boldsymbol{\alpha}_{\mathrm{r}}\left(\phi_{k}\right)$, one can always find a $\mathbf{r}_{k}$ fulfilling $\left|\mathbf{r}_{k}^{T} \boldsymbol{\alpha}_{\mathrm{r}}\left(\phi_{k}\right)\right|^{2}=N$. Hence, (13) is equivalent to

$$
\left|\boldsymbol{\alpha}_{\mathrm{t}}^{T}\left(\theta_{k_{1}}\right) \mathbf{b}\right|^{2}=\left|\boldsymbol{\alpha}_{\mathrm{t}}^{T}\left(\theta_{k_{2}}\right) \mathbf{b}\right|^{2}, \forall k_{1} \neq k_{2}
$$

Let's form a $(K) \times(N)$ matrix $\mathbf{A}$

$$
\mathbf{A} \triangleq\left[\boldsymbol{\alpha}_{\mathrm{t}}\left(\theta_{0}\right), \ldots, \boldsymbol{\alpha}_{\mathrm{t}}\left(\theta_{K-1}\right)\right]^{T}
$$

and a Hermitian matrix

$$
\Psi \triangleq \mathbf{A} \mathbf{b b}^{H} \mathbf{A}^{H}
$$

The diagonal entry of $\boldsymbol{\Psi}$ gives: $\boldsymbol{\Psi}(k, k)=\left|\boldsymbol{\alpha}_{\mathrm{t}}^{T}\left(\theta_{k}\right) \mathbf{b}\right|^{2}$. Eqn. (11) imposes $\boldsymbol{\Psi}(k, k)$ to be identical for all $k \in$ $[0, K-1]$, and in this case we have

$$
\mathbf{A b}=\sqrt{\lambda} \mathbf{u}
$$

where $\lambda$ is the singular value of $\boldsymbol{\Psi}$, and $\mathbf{u}=$ $\left[u_{0}, \ldots, u_{K-1}\right]^{T}$ the singular vector corresponding to $\lambda$ with $\left|u_{k}\right|=1, \forall k$. Hence, we can conclude:

Lemma 1. Suppose the multicast channel forming the matrix A. If there exists such $a \lambda$ and $\mathbf{u}$ fulfilling the condition (16), then it is possible to find a beamforming vector $\mathbf{b}$ that ensures identical SNR at all receivers.

Lemma 1 gives the feasibility condition for finding the beamforming vector $\mathbf{b}$. However, the optimum solution to (16) is not easy to find in its general form. Nevertheless, it is not hard to find $\mathbf{b}$ for some special cases.

Lemma 2. Suppose each row of $\mathbf{A}$ to be a normalized IDFT/DFT vector with $\mathbf{A} \mathbf{A}^{H}=\mathbf{I}$ (identity matrix). A sufficient condition for $\mathbf{b}$ to fulfill the condition (16) is

$$
\begin{aligned}
\mathbf{b}(k: K & :(\bar{N}-1) K+k) \\
& =\sqrt{N} \mathbf{a}_{k}^{*}(k: K:(\bar{N}-1) K+k)
\end{aligned}
$$

where $\mathbf{a}_{k}^{T}$ is the $k^{\text {th }}$ row of $\mathbf{A}$, and $[\cdot]^{*}$ is the conjugate.

The proof of Lemma 2 is rather trivial through the use of IDFT/DFT property and thus abbreviated. Our interest 
is mainly on the antenna-element grouping method suggested by Lemma 2, (17). Intuitively, when the multicast channel is a part of the normalized IDFT/DFT matrix, Lemma 2 suggests the use of antenna element with the index $(k: K:(\bar{N}-1) K+k)$ to serve the $k^{\text {th }}$ receiver. In this case, the SNR at every receiver is

$$
\begin{aligned}
\mathrm{SNR}_{k} & =\frac{\sigma_{s}^{2}}{\sigma_{v}^{2}}\left|\mathbf{r}_{k}^{T} \mathbf{H}_{k} \mathbf{b}\right|^{2} \\
& =\frac{N \sigma_{s}^{2}\left|\boldsymbol{\alpha}_{\mathrm{t}}^{T}\left(\theta_{k}\right) \mathbf{b}\right|^{2}}{\sigma_{v}^{2}}=\frac{\bar{N}^{2} \sigma_{s}^{2}}{\sigma_{v}^{2}}, \forall k,
\end{aligned}
$$

and each receiver evenly shares the transmit power (i.e. $(\bar{N}) /(N)=(1) /(K)$ of the transmit power); such refers to the inter-beam interference free case or orthogonal beam-splitting case.

\section{B. Inter-beam Interference Analysis}

A sufficient and necessary condition to form the IDFT/DFT matrix $\mathbf{A}$ is:

$$
\left|\sin \left(\theta_{k_{1}}\right)-\sin \left(\theta_{k_{2}}\right)\right| \in\left\{0, \ldots, \frac{2(N-1)}{N}\right\}, \forall k_{1} \neq k_{2}
$$

This is also the hypothesis of multiuser channel orthogonality often used for the beamspace technique [13]. When the hypothesis (20) does not hold in practice, inter-beam interference occurs. For the beamspace technique, inter-beam interference is often detrimental since beams carry uncorrelated information. However for the analogue-beam splitting technique, the interbeam interference behaves differently. More explicitly, the inter-beam interference could be either constructive or destructive.

When the multicast channel does not follow the condition specified in Lemma 2, there is a frequency-like mismatch between the real channel $\boldsymbol{\alpha}_{\mathrm{t}}\left(\theta_{k}\right)$ and $\mathbf{a}_{k}$

$$
\boldsymbol{\alpha}_{\mathbf{t}}\left(\theta_{k}\right)=\mathbf{D}\left(\boldsymbol{\omega}_{k}\right) \mathbf{a}_{k}
$$

where $\boldsymbol{\omega}_{k} \triangleq\left[1, \ldots, \exp \left(j \frac{2 \pi \omega_{k} k n}{N}\right)\right]^{T}, \mathbf{D}\left(\boldsymbol{\omega}_{k}\right)$ is the diagonal matrix with $\boldsymbol{\omega}_{k}$ in its diagonal, and $\omega_{k} \in$ $(-0.5,0.5)$ is the frequency-like mismatch. This model is mimic to the orthogonal frequency-division multiplexing (OFDM) but in the spatial domain. Using (19), the SNR at the $k_{1}^{\text {th }}$ receiver now read as

$$
\begin{aligned}
\mathrm{SNR}_{k_{1}} & =\frac{N \sigma_{s}^{2}\left|\mathbf{a}_{k_{1}}^{T} \mathbf{D}\left(\boldsymbol{\omega}_{k_{1}}\right) \mathbf{b}\right|^{2}}{\sigma_{v}^{2}} \\
& =\frac{N \sigma_{s}^{2}}{\sigma_{v}^{2}}\left|B\left(k_{1}\right)+\mathrm{IBI}\right|^{2}
\end{aligned}
$$

where $B\left(k_{1}\right)$ denotes the term for the $k_{1}^{\text {th }}$ beam with

$$
B\left(k_{1}\right)=\sum_{n=0}^{\bar{N}-1} \exp \left(\frac{j 2 \pi \omega_{k_{1}}\left(K n+k_{1}\right)}{N}\right)
$$

and IBI denotes the inter-beam interference specified by

$$
\mathrm{IBI}=\sum_{k_{2} \neq k_{1}} \underbrace{\sum_{n=0}^{\bar{N}-1} \exp \left(\frac{j 2 \pi\left(\omega_{k_{1}}-k_{2}\right)\left(K n+k_{2}\right)}{N}\right)}_{I\left(k_{2}\right)}
$$

It can be observed that the IBI term vanishes for the case of $\omega_{k_{1}}=0$. When $\omega_{k_{1}} \neq 0$, the interference term $I\left(k_{2}\right)$ can be constructive given

$$
\left|B\left(k_{1}\right)+I\left(k_{2}\right)\right|>\left|B\left(k_{1}\right)\right|
$$

or destructive given

$$
\left|B\left(k_{1}\right)+I\left(k_{2}\right)\right|<\left|B\left(k_{1}\right)\right|
$$

The triangular theorem tells us the condition for (26) to hold is

$$
\left|\angle\left(B\left(k_{1}\right) I^{*}\left(k_{2}\right)\right)\right|<\pi-\cos ^{-1}\left(\frac{\left|I\left(k_{2}\right)\right|}{2\left|B\left(k_{1}\right)\right|}\right)
$$

or otherwise (27) holds.

\section{Non-orthogonal Beam Splitting}

One of shortcomings for the orthogonal beam splitting is the energy leaking due to the presence of frequencylike mismatch, which could largely reduce $\left|B_{k}\right|$. To overcome this shortcoming, here we propose a nonorthogonal beam splitting approach.

The non-orthogonal approach shares the same idea as the orthogonal approach when performing the antennaelement grouping. The major difference lies in the form of the beamforming vector. Instead of using (17), the beamforming vector is now formed by

$$
\begin{aligned}
\mathbf{b}(k: K & :(\bar{N}-1) K+k) \\
& =\sqrt{N} \boldsymbol{\alpha}_{t, k}^{*}(k: K:(\bar{N}-1) K+k)
\end{aligned}
$$

where $\boldsymbol{\alpha}_{\mathrm{t}, k}$ is the concise version of $\boldsymbol{\alpha}_{\mathrm{t}}\left(\theta_{k}\right)$. By this means, we always have

$$
B_{k}=(\bar{N}) /(\sqrt{N}), \forall k
$$

which reaches the maximum. The inter-beam interference is now represented by

$$
I\left(k_{2}\right)=\sum_{n=0}^{\bar{N}-1} \exp \left(\frac{j \pi\left(\sin \theta_{k_{1}}-\sin \theta_{k_{2}}\right)\left(K n+k_{2}\right)}{N}\right)
$$

Due to $\angle B_{k}=0,(28)$ indicates that $I\left(k_{2}\right)$ is constructive for $\left|\angle\left(I\left(k_{2}\right)\right)\right|<\pi-\cos ^{-1}\left(\frac{\left|I\left(k_{2}\right)\right|}{2\left|B\left(k_{1}\right)\right|}\right)$; or destructive otherwise.

It is not hard to understand that the non-orthogonal approach outperforms the orthogonal approach thanks to the minimization of energy leaking. On the other hand, it also faces the problem of destructive interference, and this issue will be further discussed in Sec. IV. 


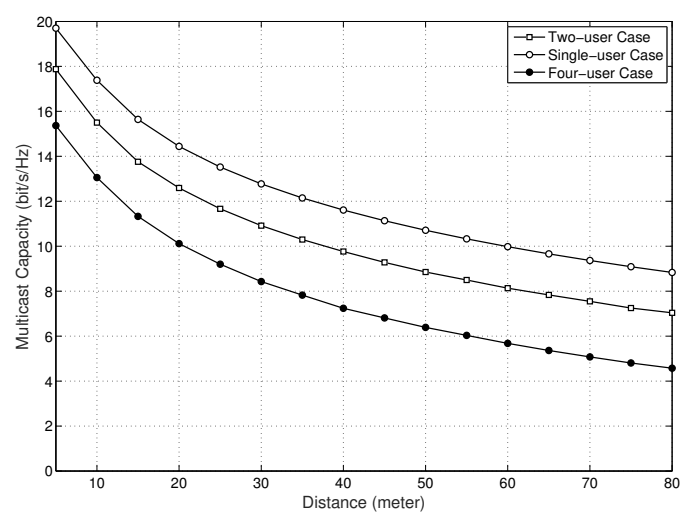

Fig. 2. Multicast channel capacity vs the radio coverage.

\section{Numerical Results AND Discussion}

Computer simulation is performed to examine the mmWave multicast channel capacity and radio coverage as far as the non-orthogonal analogue-beam splitting approach is concerned. Throughout the simulation, we assume that mobile devices operate at $26 \mathrm{GHz}$ carrier frequency with the signal bandwidth of $20 \mathrm{MHz}$. Each mobile device has 32 antenna elements supported by a single RF chain for the signal transmission or reception. The transmit power is set to $10 \mathrm{dBm}$, and the noise spectrum density is $-174 \mathrm{dBm} / \mathrm{Hz}$ [14]. For the sake of link-level performance evaluation, the system setup follows the 2-D model depicted in Fig. 1. All receivers are uniformly distributed on the circle centered at the transmitter with the radius $d$ varying from $5-80 \mathrm{~m}$. The mmWave multipath channel model is based upon the practical measurement result obtained at one of Surrey University's buildings which has a large open-plan office $\left(40 \times 40 \mathrm{~m}^{2}\right)$. Basically, the beams follow Laplacian distribution, and the multipath components are $15-30$ $\mathrm{dB}$ weaker than the LOS path. This model is well in line with other published mmWave channel models [15].

Fig. 2 illustrates the multicast channel capacity as a function of the radio coverage (i.e. $d$ in meter). Generally, the multicast channel capacity degrades with the increase of receivers. This phenomenon well coincides with our theoretical analysis in (10). Moreover, the capacity degradation due to mmWave path-loss can also be observed from the figure.

Fig. 3 illustrates the multicast channel capacity vs. the radio coverage for the case of having four receivers. The baseline for performance comparison is mainly the TDD beam-steering approach. It can be observed that the proposed beam-splitting approach significantly outperforms the TDD beam-steering approach by $2-3$ fold in the capacity given the same coverage. This is because the TDD beam-steering approach has its capacity degrades linearly with the number of receivers,

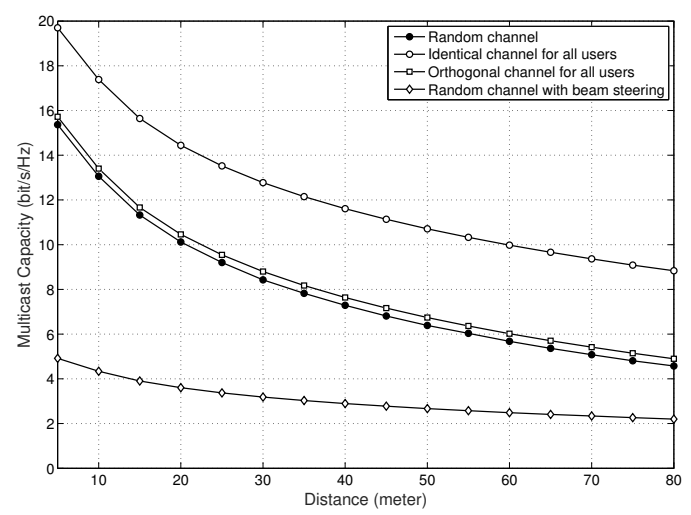

Fig. 3. Performance comparison for four-receiver mmWave multicast channel

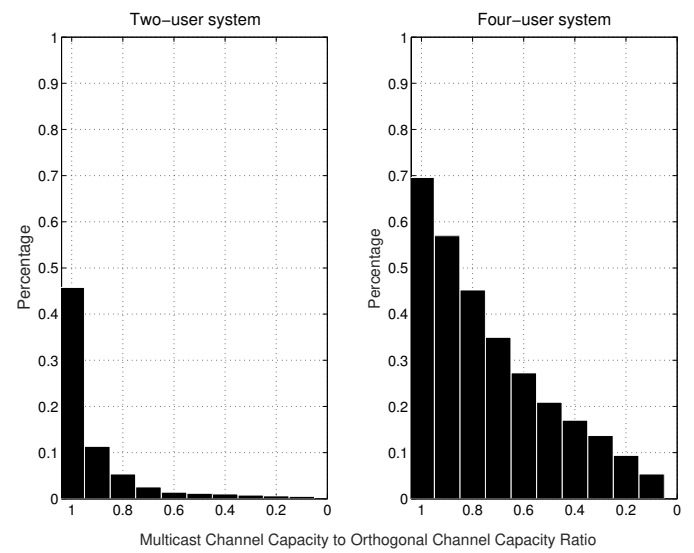

Fig. 4. Inter-beam interference characteristics for the mmWave multicast channel.

while the beam splitting approach has its capacity degrades logarithmically. When the mmWave channel is forced to be orthogonal between receivers, the nonorthogonal approach reduces to the orthogonal approach and does not suffer inter-beam interference. Interestingly, the performance of interference-free case does not offer considerable gain when comparing with the case of non-orthogonal random channel. To understand this phenomenon, we plot in Fig. 4 the percentage for the following case

$$
\frac{\text { Capacity of non-orthogonal channel }}{\text { Capacity of orthogonal channel }}=\eta \leq 1
$$

It is observed that: 1) for the two-receiver system, there are around $45 \%$ channel realizations having the capacity lower than the orthogonal-channel case. In other words, there are around $55 \%$ channel realizations offering constructive inter-beam interference. In addition, there are only a very small portion of destructive interference that largely reduces the capacity (e.g. it is around $5 \%$ for $\eta \leq 0.8)$; 2) with the increase of receivers to four, 
there are more destructive impact from the interference. Nevertheless, the portion of very destructive interference is still around $30-40 \%$, and the portion of constructive interference is more than $30 \%$. This explains the minor difference between the orthogonal channel and the random channel. Indeed, the interference will become more destructive with further increase the number of receivers. However, we argue that the channel capacity and radio coverage will have a significant drop in this case, and no longer favorable for multiuser wireless communications.

\section{CONCLUSION}

This paper has presented a novel analogue-beam splitting approach to enable D2D multicast communication over mmWave channel. Based on the 2-D circular system model, the multicast channel capacity, radio coverage as well as their trade-off were theoretically studied at the link level. The feasibility condition and design principle for the analogue-beam splitting were mathematically established. Computer simulations were utilized to elaborate our theoretical analysis and demonstrate significant gain ( $2-3$ fold) of using the proposed approach over the TDD beam-steering approach.

\section{ACKNOWLEDGMENT}

This work was partially funded by The $5 \mathrm{G}$ Innovation Centre (5GIC) and EU H2020 EXCITING project.

\section{REFERENCES}

[1] R. Alkurd, R. M. Shubair, and I. Abualhaol, "Survey on deviceto-device communications: Challenges and design issues," in 2014 IEEE 12th Int. New. Circuits. and Sys. Conf (NEWCAS), June 2014, pp. 361-364.

[2] A. Asadi, Q. Wang, and V. Mancuso, "A survey on deviceto-device communication in cellular networks," IEEE Commun. Surveys Tuts., vol. 16, no. 4, pp. 1801-1819, Fourthquarter 2014.
[3] Z. Guizani and N. Hamdi, "mmWave E-band D2D communications for 5G-underlay networks: Effect of power allocation on D2D and cellular users throughputs," in 2016 IEEE Symposium. Comput. and Commun (ISCC), June 2016, pp. 114-118.

[4] S. Wu, R. Atat, N. Mastronarde, and L. Liu, "Improving the coverage and spectral efficiency of millimeter-wave cellular networks using device-to-device relays," IEEE Trans. Commun., vol. PP, no. 99, pp. 1-1, 2017.

[5] J. Deng, O. Tirkkonen, R. Freij-Hollanti, T. Chen, and N. Nikaein, "Resource allocation and interference management for opportunistic relaying in integrated mmWave/sub-6 GHz 5G networks," IEEE Commun. Mag., vol. 55, no. 6, pp. 94-101, 2017.

[6] R. Taori and A. Sridharan, "Point-to-multipoint in-band mmWave backhaul for 5G networks," IEEE Commun. Mag., vol. 53, no. 1, pp. 195-201, January 2015.

[7] R. W. Heath, N. Gonzlez-Prelcic, S. Rangan, W. Roh, and A. M. Sayeed, "An overview of signal processing techniques for millimeter wave MIMO systems," IEEE J. Sel. Topics Signal Process., vol. 10, no. 3, pp. 436-453, April 2016.

[8] R. Bonjour, M. Singleton, S. A. Gebrewold, Y. Salamin, F. C. Abrecht, B. Baeuerle, A. Josten, P. Leuchtmann, C. Hafner, and J. Leuthold, "Ultra-fast millimeter wave beam steering," IEEE $J$. Quantum Electron., vol. 52, no. 1, pp. 1-8, Jan 2016.

[9] F. Sohrabi and W. Yu, "Hybrid beamforming with finiteresolution phase shifters for large-scale MIMO systems," in 2015 IEEE 16th Int. Workshop. Signal. Process. Advances. Wireless. Commun (SPAWC), June 2015, pp. 136-140.

[10] J. Huang, Z. Cheng, E. Chen, and M. Tao, "Low-complexity hybrid analog/digital beamforming for multicast transmission in mmWave systems," in 2017 IEEE Int. Conf. Commun (ICC), May 2017, pp. 1-6.

[11] A. Alkhateeb, O. E. Ayach, G. Leus, and R. W. Heath, "Channel estimation and hybrid precoding for millimeter wave cellular systems," IEEE J. Sel. Topics Signal Process., vol. 8, no. 5, pp. 831-846, Oct 2014.

[12] J. Hou, Y. Ma, N. Yi, and R. Tafazolli, "Reduced-complexity coordinated beamforming for multicell downlink max-min SINR problem," IEEE Wireless Commun. Lett., vol. 3, no. 4, pp. 353356, Aug 2014.

[13] A. Sayeed and J. Brady, "Beamspace mimo for high-dimensional multiuser communication at millimeter-wave frequencies," in 2013 IEEE GLOBECOM, Dec 2013, pp. 3679-3684.

[14] A. Goldsmith, Wireless Communications. New York, NY, USA: Cambridge University Press, 2005.

[15] "White paper on 5G Channel Model for bands up to100 GHz," 5GCHFB in conjunction with IEEE Globecom17, Tech. Rep., Dec 2017. 